\title{
Immunisation coverage in the rural Eastern Cape - are we getting the basics of primary care right? Results from a longitudinal prospective cohort study
}

\author{
K le Roux, ${ }^{1} \mathrm{MB}$ ChB, MMed Sci; O Akin-Olugbade, ${ }^{2} \mathrm{BA}$; L S Katzen, ${ }^{3}$ BSc, MSc; C Laurenzi, ${ }^{4}$ BA, MSc; N Mercer, ${ }^{5}$ MS; \\ M Tomlinson, ${ }^{6} \mathrm{PhD} ; \mathrm{M}$ J Rotheram-Borus, ${ }^{7} \mathrm{PhD}$ \\ ${ }^{1}$ Visiting research scholar, Center for Health and Wellbeing, Woodrow Wilson School, Princeton University, New Jersey, USA (at time of study); \\ Primary Healthcare Directorate, University of Cape Town, South Africa; Zithulele Hospital, Eastern Cape, South Africa \\ ${ }^{2}$ Undergraduate student at Princeton University, New Jersey, USA (at time of study) \\ ${ }^{3}$ Philani Centres Nutrition Trust, Khayelitsha, Cape Town, South Africa \\ ${ }^{4}$ Prevention Research for Community, Family and Child Health, Stellenbosch University, Cape Town, South Africa \\ ${ }^{5}$ Center for HIV Identification, Prevention, and Treatment Services (CHIPTS), University of California, Los Angeles, USA (at time of study) \\ ${ }^{6}$ Department of Psychology, Stellenbosch University, Cape Town, South Africa \\ ${ }^{7}$ Department of Psychiatry, Semel Institute, University of California, Los Angeles, USA
}

Corresponding author: Kle Roux (karlleroux@gmail.com)

\begin{abstract}
Background. Immunisations are one of the most cost-effective public health interventions available and South Africa (SA) has implemented a comprehensive immunisation schedule. However, there is disagreement about the level of immunisation coverage in the country and few studies document the immunisation coverage in rural areas.

Objective. To examine the successful and timely delivery of immunisations to children during the first 2 years of life in a deeply rural part of the Eastern Cape Province of SA.

Methods. From January to April 2013, a cohort of sequential births $(N=470)$ in the area surrounding Zithulele Hospital in the OR Tambo District of the Eastern Cape was recruited and followed up at home at 3, 6, 9, 12 and 24 months post birth, up to May 2015. Immunisation coverage was determined using Road-to-Health cards.

Results. The percentages of children with all immunisations up to date at the time of interview were: $48.6 \%$ at 3 months, $73.3 \%$ at 6 months, $83.9 \%$ at 9 months, $73.3 \%$ at 12 months and $73.2 \%$ at 24 months. Incomplete immunisations were attributed to stock-outs (56\%), lack of awareness of the immunisation schedule or of missed immunisations by the mother (16\%) and lack of clinic attendance by the mother (19\%). Of the mothers who had visited the clinic for baby immunisations, $49.8 \%$ had to make multiple visits because of stock-outs. Measles coverage (of at least one dose) was $85.2 \%$ at 1 year and $96.3 \%$ by 2 years, but $20.6 \%$ of babies had not received a second measles dose (due at 18 months) by 2 years. Immunisations were often given late, particularly the 14-week immunisations.

Conclusions. Immunisation rates in the rural Eastern Cape are well below government targets and indicate inadequate provision of basic primary care. Stock-outs of basic childhood immunisations are common and are, according to mothers, the main reason for their children's immunisations not being up to date. There is still much work to be done to ensure that the basics of disease prevention are being delivered at rural clinics in the Eastern Cape, despite attempts to re-engineer primary healthcare in SA.
\end{abstract}

S Afr Med J 2017;107(1):52-55. DOI:10.7196/SAMJ.2017.v107i1.11242

Immunisation coverage of children is a cornerstone of primary healthcare (PHC) and an important gauge of the quality of the health service in a country, ${ }^{[1]}$ but is also a good indicator of how far a country is from preventable disease outbreaks, such as the measles outbreak that occurred in South Africa (SA) in 2010. ${ }^{[2]}$

SA's immunisation schedule has been significantly expanded since 1994, with the recent addition of the pneumococcal (PCV) and rotavirus (RV) vaccines in $2009,{ }^{[3]}$ and is the most comprehensive in Africa ${ }^{[4]}$ As part of the 2010 'Re-engineering of Primary Health Care' policy, the South African National Department of Health (NDoH) has emphasised the importance of doing the basics of PHC right. ${ }^{\left[{ }^{[}\right]}$ However, there has been disagreement about how well the schedule has been implemented on the ground and the $\mathrm{NDoH}$ has disputed low WHO/UNICEF immunisation coverage figures. ${ }^{[3,6]}$ After the 2011 census provided revised estimates of the number of children under the age of 1 year, the $\mathrm{NDoH}$ immunisation indicators were adjusted downwards, but were still significantly higher that WHO/UNICEF estimates. $^{[4,7]}$
The quality of $\mathrm{NDoH}$ immunisation data gathered through the District Health Information System (DHIS) is poor ${ }^{[6]}$ and apart from one study, ${ }^{\left[{ }^{[8]}\right.}$ there are limited data on immunisation coverage in rural areas of SA and, to the best of our knowledge, nothing about coverage in rural Eastern Cape.

In this study we assessed immunisation coverage of the South African Expanded Programme of Immunisations (EPI-SA) during the first 2 years of life and the timeliness of immunisations in the first year of life. The study formed part of the Zithulele Births Follow up Study (ZiBFUS), a prospective, longitudinal cohort study, initiated in January 2013.

\section{Methods}

Setting

Zithulele Hospital is a 146-bed district hospital, serving a catchment area with a population of approximately $130000^{[9]}$ and fourteen clinics, and is situated in the King Sabata Dalindyebo (KSD) sub-district of the OR Tambo District in the Eastern Cape, a National Health Insurance 
(NHI) pilot district. It is one of the two poorest municipalities in SA. ${ }^{[10]}$ There has been a sustained and significant improvement in the quality of healthcare delivered at Zithulele Hospital and its feeder clinics since $2005 .^{[9,11,12]}$ Outpatient numbers and in-facility deliveries at Zithulele Hospital tripled between July 2005 and July 2015, while perinatal mortality rates decreased from 42 per 1000 live births to below 20 per 1000 , paediatric in-hospital mortality decreased from $10 \%$ to 2.5\% and more than 5000 patients were started on ARVs (personal communication - C B Gaunt, clinical manager, Zithulele Hospital, 27 October 2015).

The hospital clinical team also invested significantly in 10 PHC feeder clinics, by sending doctors, occupational therapists, physiotherapists, dentists, dieticians, speech therapists and audiologists to these clinics on a regular rotation. As a result, the level of support received by PHC clinics in the Zithulele Hospital catchment area is better than in most rural areas of SA.

\section{Sample}

We recruited a cohort at birth from Zithulele Hospital and the area covered by its closest PHC clinics between 14 January 2013 and 15 April 2013. All women who gave birth at Zithulele Hospital, at one of the ten closest clinics, on the way to a health facility, or at home in the area covered by the clinics during this time period were included in the study. Women who travelled to the hospital from outside this catchment area to give birth at the hospital were excluded, because of lack of resources for regular follow-up of women further afield. Informed consent was obtained from all women; in cases where a participant was under 18 years of age, informed consent was obtained from her and a parent or guardian as well. Ethical approval was obtained from the Health Research Ethics Committee at Stellenbosch University (N12/08/046) and permission for the recruitment to occur in government health facilities was granted by the Eastern Cape Department of Health through the Office of Epidemiological Research and Surveillance Management.

Most women were recruited from the maternity ward of Zithulele Hospital, where a field worker (FW) was stationed every day of the week (including weekends). To identify women who gave birth at home or in one of the feeder clinics, the research team relied on nurses at the clinics to identify these cases, and offered nurses a small airtime reimbursement to contact the FWs with the woman's telephone number. The principal investigator, having previously worked as a medical officer in all of the feeder clinics, had built good relationships with the nurses there over many years. Links were also established with traditional leaders in the community, with the hope that they could help us to identify mothers giving birth at home. The majority of home births were referred to us by nurses at the time of the mother's first visit to the clinic. We believe that virtually all home births during the recruitment period were identified in this way because of the strong incentive for mothers to visit a clinic or hospital soon after birth to acquire a Road-to-Health card (RtHC) for their baby. Not only is the RtHC a type of health 'passport' and an extremely important health record, but it is also used to apply for a child support grant (R250 per month in 2013) from the South African Social Security Agency (SASSA). ${ }^{[13]}$

FWs were recruited locally and were trained by experienced facilitators from Stellenbosch University for a period of 6 weeks. Interview techniques, the use of cell phones as a data collection tool, research ethics, and confidentiality were covered, and the birth questionnaire was piloted in the field. For each of the subsequent surveys, in-house training and piloting was performed. Questionnaires were initially written in English, and then translated into isiXhosa by the FWs, who are all proficient in English and familiar with the type of isiXhosa spoken in the part of the rural Eastern Cape where the study was performed. Questionnaires were then loaded onto cell phones using the programme Mobenzi Researcher, ${ }^{[14]}$ and all data entered into the Mobenzi Researcher platform in the field were checked by the research co-ordinator or on-site principal investigator in the presence of the relevant data capturer within a few days of entry. All interviews were voice-recorded, and an experienced isiXhosa-speaking researcher at Stellenbosch University reviewed a select number of interviews per FW each month, for further quality control and verification of the data's accuracy. The project co-ordinator also conducted field visits on a weekly basis for the first year to further ensure the quality of data collected.

Birth interviews were usually performed within $1-2$ days of birth at the hospital, and within $1-2$ weeks if the child was born at home or at the clinic. Mother-infant pairs were then followed up at 3, 6, 9, 12 and 24 months. Ninety-two percent of follow-up interviews were conducted at the child's home, while the rest were performed at another convenient location for the mother, such as a clinic, trading store or the hospital.

Immunisation status was checked at each follow-up visit, and FWs assessed whether all immunisations that should have been given by the relevant age had indeed been given. (For the immunisations checked at each interview, see Table 2.) If a single immunisation was missing, a child's immunisations were classified as incomplete. If the mother or caregiver gave consent, the FW took a photo of the immunisation page in the RtHC, which allowed for confirmation of the immunisation status at each visit, and also enabled us to assess whether immunisations had been given at the correct time, as the date each immunisation is administered is noted on the card by the clinic nurse.

Participants who did not have RtHCs were not included in the assessment of immunisation status. At the 3-and 6-month interviews, mothers and caregivers who had been to the clinic were asked whether they had been instructed to return after a routine (immunisation) visit because of the unavailability of one or more immunisations. Furthermore, if the child's immunisations were incomplete, mothers and caregivers were asked why this was the case.

Data were analysed using SAS version 9.4 (SAS, USA).

\section{Results}

A total of 493 women fulfilled the inclusion criteria. Of this sample, 23 women (5\%) refused or were unable to provide informed consent as a result of intellectual impairment, hearing impairment or psychiatric illness, and were therefore excluded from the study. There were nine sets of twins, but the second twin was excluded from this analysis, and therefore 470 mother-infant pairs were evaluated. The age range of the women was from 14 to 52 , and 77 of the women were under 18 years old. The HIV prevalence rate of $28.5 \%$ was slightly lower than the prevalence rate of $29.8 \%$ for the OR Tambo District in the 2012 National Antenatal Sentinel HIV Prevalence Survey (76\% of women in the study who remembered their antenatal care initiation date initiated antenatal care in 2012, which is when blood for syphilis and the Sentinel HIV Prevalence Survey would have been taken). ${ }^{[15]}$

The women enrolled into the study reflect a poor rural population with little access to municipal services such as electricity and water (Table 1). While $56 \%$ had passed grade 9 , only $7 \%$ had completed 12 years of schooling, $1 \%$ had a post-school qualification and nearly $5 \%$ of mothers had never attended school. Over $90 \%$ of households received some kind of government grant at the time of the birth survey. Less than a fifth of households had access to electricity at baseline, with two-thirds using wood as their primary fuel source. Only $30 \%$ had access to a communal water tap, with $48 \%$ relying on unsafe river water. At the 2-year follow-up, $36 \%$ of mothers had access to communal taps, $27 \%$ had access to mains electricity, and another $7 \%$ had gained access to government-supplied solar panels, indicating a modest extension of municipal services in the area surveyed. The vast majority of births were in a health facility $(86 \%)$, and of those who delivered at home, only one woman had intended to do so. By 2 years, 
$34 \%$ of the children were being primarily cared for by someone other than their mother, with maternal grandmothers caring for these children in 55\% of cases; this shift was mostly as a result of mothers returning to school or work.

Follow-up rates were between 84.7 and $92.1 \%$, and were $88 \%$ at 2 years (Table 2). Twelve-month follow-up rates were bolstered by the 2014 platinum mining strike, which started in January and lasted 5 months. During this strike, most men employed in the mines (and also the mother-infant pairs participating in the study) were back home from the North West Province. Twenty-two infants died in the first year, two of whom were the second twin and are therefore not included in this analysis. No deaths were recorded the second year of the study.

A high proportion (between 89 and 96\%) of mothers or caregivers had the children's RtHC available at the interview (Table 2), and at 2 years only $2 \%$ reported that the child's RtHC had been either lost or destroyed. Immunisation rates at the 3-month interview were less

\begin{tabular}{ll} 
Table 1. Descriptors of a consecutive series of women giving \\
birth in the rural Eastern Cape and their babies $(\mathbf{N}=\mathbf{4 7 0})$ \\
\hline Demographic characteristics (mother) \\
\hline Age, mean (SD) & $24.9(7.03)$ \\
Highest education level attained (in grade), mean & $8.19(2.98)$ \\
(SD) & \\
Household size before arrival of new baby, median & $7(4)$ \\
(IQR) & \\
Primiparous, \% & 39 \\
HIV-positive, \% & 29 \\
Mother lives with father of child, \% & 18 \\
Electricity in home, \% & 16 \\
Water source, \% & \\
River water & 48 \\
Communal tap & 27 \\
Water tank & 16 \\
Well & 9 \\
Employment status, \% & \\
Employed & 4 \\
Schooling & 16 \\
Unemployed & 80 \\
Baby characteristics & \\
Sex (female), \% & 44 \\
Birth-weight, mean $z$-score (SD) & $-0.73(1.21)$ \\
Low birth weight (<2 500 g), \% & 16 \\
Place born, \% & \\
At home & 10 \\
On way to health facility & 4 \\
At community health centre & 9 \\
At hospital & \\
SD = standard deviation; IQR = interquartile range. & \\
& \\
\hline
\end{tabular}

than $50 \%$, but this had improved by 6 and 9 months (Table 2). At 12 months, the fully immunised group was again smaller, because some of the children in the study were missing either their measles containing vaccine 1 (MCV 1 ) or pneumococcal conjugate vaccine 3 (PCV 3) (or both), which is due at 9 months. MVC 1 had an $85 \%$ coverage rate at 1 year and MCV 2 a $79.4 \%$ coverage rate at 2 years. Just less than $4 \%$ of children had received neither MCV1 nor MVC2 by the time of the 2-year interview.

Fifty-six percent of women whose children did not have all their immunisations up to date blamed stock-outs at the clinic (51.9\% at 3 months and $62.6 \%$ at 6 months), $19 \%$ said that they had not gone to the clinic yet, while $16 \%$ said that they were unaware of the fact that the immunisations were incomplete. In these cases, some participants went for immunisations and were given one, but not all, of the required immunisations for the relevant date, but were not informed that the immunisations were incomplete; others were simply unaware that they needed to go for further immunisations. By 6 months, $49.8 \%$ of women in the study who had gone for immunisations, including those whose children had all immunisations up to date at the time of the interview, reported that they had to return to the clinic for an immunisation that was out of stock at least once.

Finally, as can be seen in Fig. 1, a significant proportion of immunisations were administered more than 2 weeks after they were due. This trend was most prominent at 14 weeks, when between $60 \%$

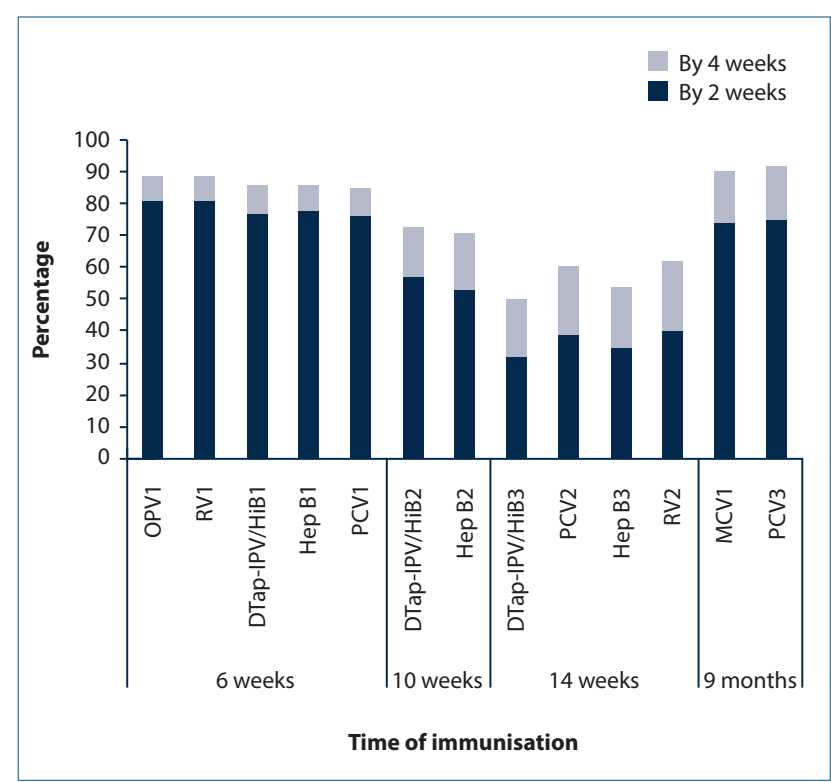

Fig. 1. Percentage of immunisations that were given on time (within 2 weeks and 4 weeks of expected date) at 6 weeks, 10 weeks, 14 weeks and 9 months. $(O P V=$ oral polio vaccine; $R V=$ rotavirus vaccine; DTap-IPV/ $H i B=$ diptheria, tetanus, acellular pertussis, inactivated polio vaccine and Haemophilus influenzae type $B$ combined; Hep $B=$ hepatitis $B$ vaccine; $P C V=$ pneumococcal conjugate vaccine; $M C V=$ measles-containing vaccine. $)$

Table 2. Follow-up and immunisation rates at the 3-, 6-, 9-, 12- and 24-month interviews

\begin{tabular}{|c|c|c|c|c|c|}
\hline & 3 months & 6 months & 9 months & 12 months & 24 months \\
\hline Follow-up rate, $\%(n / N)$ & $84.8(390 / 460)$ & $92.1(420 / 456)$ & $88.3(401 / 454)$ & $91.3(411 / 450)$ & $88.0(396 / 450)$ \\
\hline RtHC available at interview, \% $(n / N)$ & $96.4(376 / 390)$ & $95.5(401 / 420)$ & $91.3(366 / 401)$ & $89.5(368 / 411)$ & $89.4(354 / 396)$ \\
\hline $\begin{array}{l}\text { All immunisations up to date at interview, } \\
\%(n / N)\end{array}$ & $48.6(175 / 360)$ & $73.3(294 / 401)$ & $83.9(307 / 366)$ & $73.3(269 / 367)$ & $73.2(259 / 354)$ \\
\hline Deaths during time period, $n$ & 10 & 4 & 2 & 4 & 0 \\
\hline Immunisations assessed at interview & $\begin{array}{l}\text { Birth, } 6 \mathrm{wk}, \\
10 \mathrm{wk}\end{array}$ & $\begin{array}{l}\text { Birth, } 6 \text { wk, } \\
10 \mathrm{wk}, 14 \mathrm{wk}\end{array}$ & $\begin{array}{l}\text { Birth, } 6 \text { wk, } \\
10 \mathrm{wk}, 14 \mathrm{wk}\end{array}$ & $\begin{array}{l}\text { Birth, } 6 \text { wk, } 10 \text { wk, } \\
14 \text { wk, } 9 \text { mo }\end{array}$ & $\begin{array}{l}\text { Birth, } 6 \text { wk, } 10 \text { wk, } \\
14 \text { wk, } 9 \text { mo, } 18 \text { mo }\end{array}$ \\
\hline
\end{tabular}


and $68 \%$ of the immunisations were given more than 2 weeks after their due date, while $50 \%$ of DTap-IPV/HiB 3 immunisations were given more than 4 weeks late.

\section{Discussion}

The NDoH has set ambitious targets for immunisation rates in the Health Ministry's Annual Performance Plan 2014/2015 - 2016/7, with the stated aim of $95 \%$ coverage for immunisations by 1 year and $85 \%$ coverage for measles second dose (MCV2). ${ }^{[16]}$ The ZiBFUS demonstrates that in rural areas, clinics are falling short of national targets for immunisation coverage, even in an NHI pilot district with a well-functioning district hospital. The ZiBFUS data show 1-year immunisation coverage of $73.3 \%$, in comparison with the target of $95 \%$, and measles second dose coverage of $79.4 \%$, as compared with the $85 \%$ target. It is encouraging that $96.3 \%$ of babies had received at least one measles immunisation by 2 years. However, MCV 1 immunisation coverage still falls well below the NDoH target of $95 \%$ at 1 year, which is also the level required for the development of herd immunity. ${ }^{[17]}$ It is important to note that we were only able to assess immunisation status on children who had RtHCs available. Although RtHC availability was high $-96.4 \%$ at 3 months and $89.4 \%$ at 24 months - we are probably underestimating immunisation coverage slightly, as children without RtHCs are less likely to have been immunised, as clinic nurses require them for vaccinations to be given.

As a point of comparison, and not unexpectedly for an underserved rural area, our figures for 1-year immunisation coverage are lower than the NDoH national estimates for 2012/13, which vary between 94\% (Annual Performance Plan) ${ }^{[16]}$ and 83.4\% (2014/15 District Health Barometer). ${ }^{[3]}$ Interestingly, our figures are higher than WHO/UNICEF national estimates for $2012 / 3$, which indicate that immunisation coverage was around $69 \% \cdot{ }^{[7]}$

In light of the prevention imperative of immunisations and the risk to herd immunity due to poor coverage, it is notable that stockouts of immunisations had affected nearly $50 \%$ of the all the women and children participating in the study by the 6 -month interview, necessitating one or more subsequent visits to ensure adequate coverage. Furthermore, $56 \%$ of women whose children did not have up-to-date immunisations attributed this to stock-outs, while only $19 \%$ of women indicated that they had not yet gone to their clinic for the required routine vaccinations. There may be some reporting bias here, in that women might try to justify their children's incomplete immunisations by blaming nurses or clinics. However, the fact that $45 \%$ of the women who said they had been asked to return to the clinic at least once had children whose immunisations were all up to date indicates that stock-outs are not simply being used as an excuse, but that this is indeed an extensive problem. There is a tendency for healthcare workers to blame mothers for incomplete immunisations, ${ }^{[18]}$ but our data indicate that nearly three-quarters of missed immunisations were due to a health system failure - either a stock-out (56\%) or lack of information to women about their baby's immunisations being incomplete (16\%).

Furthermore, the knock-on effect of stock-outs, which delay routine immunisations and therefore cause other scheduled immunisations to also be delayed, is well demonstrated, with 38 - $50 \%$ of the 14 -week immunisations given more than 1 month late. This finding, coupled with the difficulty that many mothers have in accessing rural clinics, means that young infants are left potentially vulnerable to dangerous preventable diseases, and resultant malnutrition and developmental delay. ${ }^{[19]}$

Our data cannot be generalised to the whole of SA, but gives an indication of the immunisation coverage and timeliness of routine childhood immunisations in poor rural communities. It is clear that, despite SA's impressive immunisation policies and the emphasis on the basics of PHC under the 'Re-engineering of Primary Health Care' strategy, immunisations are not always available to infants in the rural Eastern Cape. This places an unnecessary burden on rural women and their children and puts poor rural communities at risk of preventable disease outbreaks.

Acknowledgements. The authors declare no conflicts of interest. Funding for the study was provided by the ELMA Foundation through a grant to Philani Centres Nutrition Trust. MT is a lead investigator with the Centre of Excellence in Human Development, University of the Witwatersrand, SA and is supported by the National Research Foundation, SA.

1. Burton A, Monasch R, Lautenbach B. WHO and UNICEF estimates of national infant immunization coverage: Methods and processes. Bull World Health Organ 2009;87(7):535-541. http://dx.doi org/10.2471/BLT.08.053819

2. Le Roux DM, Le Roux SM, Nuttall JJ, Eley BS. South African measles outbreak $2009-2010$ as experienced by a paediatric hospital. S Afr Med J 2012;102(9):760-764.

3. Bamford L. Immunisations (Chapter 7). In: Massyn N, Peer N, Padarath A, Barron P, Day C, eds. District Health Barometer 2014/15. Durban: Health Systems Trust, 2015: 128-139. http://www.hst.org. District Health Barometer 2014/15. Durban: Health Systems Trust, 2015: 128-139. http://www.hst.org. za/publications/district-health-barometer-201415-1 (accessed 4 December 2016.

Wiysonge CS, Ngcobo NJ, Jeena PM, et al. Advances in childhood immunisation in South Africa: Where to now? Programme managers' views and evidence from systematic reviews. BMC Public Health 2012;12:578. http://dx.doi.org/10.1186/1471-2458-12-578

5. Barron P, Shasha W, Schneider H, et al. Re-engineering of Primary Health Care in South Africa. Discussion document. Department of Health, 2010. http://docslide.us/documents/draft-2010document-on-the-re-engineering-of-primary-health-care-in-south-africa.html (accessed 27 June 2016).

6. Bateman C. Vaccines: SA's immunisation programme debunked. S Afr Med J 2016;106(4):318-319. http://dx.doi.org/10.7196/SAMJ.2016.v106i4.10765

UNICEF. Country Statistics: South Africa. UNICEF. http://www.unicef.org/infobycountry/southafrica statistics.html (accessed 4 May 2016).

8. Fadnes LT, Jackson D, Engebretsen IM, et al. Vaccination coverage and timeliness in three South African areas: A prospective study. BMC Public Health 2011;11:404. http://dx.doi.org/10.1186/14712458-11-404

9. Baleta A. Rural hospital beats the odds in South Africa. Lancet 2009;374(9692):771-772. http://dx.doi. org/10.1016/S0140-6736(09)61577-4 10. Deprivation index shows Alfred Nzo municipality is the worst in SA. Business Day Live. 7 June 2016.
http://www.bdlive.co.za/national/2016/06/07/deprivation-index-shows-alfred-nzo-municipality-is-theworst-in-sa (accessed 27 June 2016).

11. Gaunt CB. Are we winning? Improving perinatal outcomes at a deeply rural district hospital in South Africa. S Afr Med J 2010;100(2):101-104.

12. Young C, Gaunt B. Providing high-quality HIV care in a deeply rural setting - the Zithulele experience. S Afr J HIV Med 2014;15(1):28-29. http://www.scielo.org.za/scielo.php?script=sci_abstract\&pid=S207867512014000100013\&lng=en\&nrm=iso\&tlng=en (accessed 6 February 2016).

13. South African Government. Child support grant. http://www.gov.za/services/child-care-social-benefits/ child-support-grant (accessed 16 February 2016)

14. Tomlinson M, Solomon W, Singh Y, et al. The use of mobile phones as a data collection tool: A report from a household survey in South Africa. BMC Med Informatics Decision Making 2009;9(1):51. http:// dx.doi.org/10.1186/1472-6947-9-51

15. National Department of Health, South Africa. The 2012 National Antenatal Sentinel HIV and Herpes National Department of Health, South Africa. The 2012 National Antenatal Sentinel HIV and Herpes
Simplex type-2 Prevalence Survey. http://www.hst.org.za/publications/2012-national-antenatal-sentinelSimplex type-2 Prevalence Survey. http://www.hst.org.za/publications/2012-

16. National Department of Health, South Africa. Annual Performance Plan 2014/5-2016/7 - Ministry of Health. 2014. http://www.hst.org.za/publications/national-department-health-annual-performanceplan-201415-201617 (accessed 26 April 2016).

17. Fine P, Eames K, Heymann DL. 'Herd immunity’: A rough guide. Clin Infect Dis 2011;52(7):911-916. http://dx.doi.org/10.1093/cid/cir007

8. Guerra FA. Delays in immunization have potentially serious health consequences. Pediatr Drugs 2007;9:143. http://dx.doi.org/10.2165/00148581-200709030-00002

19. Salsberry PJ, Nickel JT, Mitch R. Why aren't preschoolers immunized? A comparison of parents' and providers' perceptions of the barriers to immunizations. J Comm Health Nursing 1993;10(4):213-224. http://dx.doi.org/10.1207/s15327655jchn1004_2

Accepted 29 September 2016 